\title{
Helicobacter pylori detection techniques: comparison of sensitivity, specificity and cost
}

\author{
Waidyarthne EI ${ }^{1}$, Mudduwa LKB ${ }^{1}$, Lekamwasam JDVC ${ }^{2}$, Lekamwasam ${ }^{1}$ \\ ${ }^{I}$ Faculty of Medicine, University of Ruhuna, Galle, Sri Lanka \\ ${ }^{2}$ Teaching Hospital, Karapitiya, Galle, Sri Lanka
}

Correspondence: Dr.E.I.Waidyarathne (eiwaidya@yahoo.co.uk)

\begin{abstract}
Background and Aims: In Sri Lanka, prevalence of $H$. pylori infection and the value of $H$. pylori detection techniques are not well known. We intended to find out the prevalence of $H$. pylori among a group of patients undergoing upper gastrointestinal endoscopy and to study the sensitivity and specificity of four selected diagnostic techniques.

Methods: This cross-sectional study included 251 patients who underwent routine upper gastrointestinal endoscopy at Teaching Hospital, Karapitiya, Galle. Culture, histology, urease test and serology (both anti $H$. pylori IgA and IgG) were used to diagnose $H$. pylori infection. Sensitivity and specificity of each diagnostic test were calculated taking histology as the gold standard. A particular patient was considered H. pylori positive when either Hematoxylin \& Eosin or Modified Giemsa staining revealed the morphological evidence of $H$. pylori.

Results: Histologically, the overall prevalence of H. pylori infection was $49.4 \%$. As histology was taken as the reference, both staining methods showed $100 \%$ specificity. Urease test showed a low sensitivity (32\%) and a moderate specificity (68\%) while culture and serology had very low sensitivity (7\% and 5\% respectively) but high specificity ( $97 \%$ for both). Of the four tests, histology was the most expensive test followed by serology, culture, and urease test.
\end{abstract}

Conclusions: The prevalence of $H$. pylori infection among the patients undergoing routine upper gastrointestinal endoscopy is low when compared with similar studies from other developing countries. Histology with Modified Giemsa technique appears to be the best diagnostic technique in our setup.

Key words: H. pylori, urease test, endoscopy, histology, Sri Lanka

\section{Introduction}

Prevalence of $H$. pylori infection shows a wide geographical variation. While most industrialized countries report a low prevalence, a higher prevalence has been reported from developing countries (1-3). Prevalence of $H$. pylori infection among Sri Lankans is unclear as existing data show a considerable variation (4-6). The sensitivity and specificity of $H$. pylori detection techniques vary in different settings but no such information is available in our country.
The aim of our study was to determine the sensitivity and specificity of four selected diagnostic tests; histology, urease test, serology and culture used to diagnose $H$. pylori infection and compare their direct and indirect costs.

\section{Methods}

We selected 251 consecutive patients who underwent routine upper gastrointestinal endoscopy for clinical indications in Medical and Surgical Endoscopy Units at Teaching Hospital, Karapitiya, 
Galle. Patients who had taken specific H. pylori eradication therapy during the previous six months and patients who consumed antibiotics which are active against $H$. pylori during four weeks preceding the endoscopy were excluded. Informed written consent was obtained from all the patients who were willing to participate.

We obtained nine gastric biopsies, five antral and four corporals, from each patient. One biopsy each from antrum and corpus were collected into two separate sterile containers with $0.9 \%$ physiological saline. Similarly, one biopsy each from antrum and corpus were collected into two separate $0.6 \mathrm{ml}$ eppendorf tubes containing urea broth. Remaining three antral biopsies and two corporal biopsies were collected to two separate containers with $10 \%$ formalin. Three milliliters of peripheral venous blood was obtained from each patient to bottles containing EDTA. The blood samples and culture bottles were transported to the laboratory in ice $\left(4^{0} \mathrm{C}\right)$.

Culture was performed on 62 patients. The media used was Columbia agar (CM331) with 5\% laked horse blood (SR48) supplemented with H. pylori selective supplement (SR 147) (7). The culture plates were incubated at $37^{\circ} \mathrm{C}$ for 3 days under microaerophilic $\left(5 \% \mathrm{O}_{2}, 10 \% \mathrm{CO}_{2}\right.$ and $\left.85 \% \mathrm{~N}_{2}\right)$ conditions using gas generating kits (Campy Gen CN 0025A). Colonies were identified by the colony morphology, three biochemical tests being positive i.e. urease, oxidase and catalase and by the presence of characteristic Gram negative, pale pink, slender curved bacilli by Modified Gram stain $(8,9)$.

Urease test was performed on 210 patients. The urea broth solution prepared according to the guidelines by McNulty et al (10). After immersing the biopsy specimen in urea solution the tubes were observed at one hour intervals till 24 hours for a change in color. The test was considered positive when the color of the urea solution changed from orange to magenta.

Histological identification of $H$. pylori was done in all 251 patients using both Hematoxylin and Eosin (H\&E) and Modified Giemsa staining techniques (11). Presence of $H$. pylori was confirmed when either H\&E or modified Giemsa showed the characteristic morphological evidence of H.pylori.

Out of the 251 patients, a randomly selected subgroup of 125 underwent Enzyme-Linked Immuno-Sorbent Assay (ELISA) for the detection of
anti-H. pylori IgA and IgG antibodies in plasma. Soon after the collection of blood samples plasma were separated and stored at $-20^{\circ} \mathrm{C}$. Detection of anti $H$. pylori $\operatorname{IgA}$ and anti $H$. pylori $\operatorname{IgG}$ was done with using the ELISA test kits manufactured by Human Gesellschaft fur Biochemica und Diagnostica GmbH, Germany.

Of the four diagnostic techniques, histology was considered the gold standard $(12,13)$. We considered a particular patient as $H$. pylori positive when either $\mathrm{H} \& \mathrm{E}$ or Modified Giemsa staining revealed the morphological evidence of H.pylori. The sensitivity and specificity of diagnostic methods were calculated taking histology as the reference.

The direct and indirect costs of these four diagnostic tests were calculated by adding the cost of consumables and man power. The costs for standard laboratory equipment were not taken into consideration since we performed these tests in already available laboratory settings. Cost of man power was calculated based on the monthly wages of the people involved. The estimated day salary of each person was divided by number of hours at work and calculated the salary per one hour. Average time required to perform each test was calculated and multiplied by the salary per hour and obtained the cost for man power.

The ethical clearance for the study was obtained from the Ethics Committee, Faculty of Medicine, University of Ruhuna, Sri Lanka.

\section{Results}

Culture isolated $H$. pylori from three patients. Positive urease activity was demonstrated in 67 patients either or both in antrum and corpus. Four patients became positive for IgG only while one patient became positive for both $\operatorname{IgG}$ and $\operatorname{IgA}$. Morphological evidence of $H$. pylori was present in 124 patients in either or both antrum and corpus with both H\&E and modified Giemsa stains. With H\& E alone 91 patients became positive for $H$. pylori while with Giemsa alone 101 patients became positive. Histologically the prevalence of $H$. pylori in this referred sample was $49.4 \%$

According to the gold standard the sensitivity and specificity of diagnostic methods are summarized in table 1. 
Table 1: Sensitivity and specificity of different $H$. pylori diagnostic techniques when histology (H\&E and Modified Giemsa) was taken as the gold standard.

\begin{tabular}{|c|c|c|c|c|c|c|}
\hline Test & $\begin{array}{l}\text { True } \\
\text { Positives }\end{array}$ & $\begin{array}{l}\text { True } \\
\text { Negatives }\end{array}$ & $\begin{array}{l}\text { False } \\
\text { Positives }\end{array}$ & $\begin{array}{l}\text { False } \\
\text { Negatives }\end{array}$ & Sensitivity & Specificity \\
\hline Urease test $(n=210)$ & 36 & 67 & 31 & 76 & $32 \%$ & $68 \%$ \\
\hline Serology $(n=125)$ & 3 & 63 & 2 & 57 & $5 \%$ & $97 \%$ \\
\hline Culture $(n=62)$ & 2 & 32 & 1 & 27 & $7 \%$ & $97 \%$ \\
\hline $\begin{array}{l}\mathrm{H} \& \mathrm{E}^{\dagger} \text { alone } \\
(\mathrm{n}=251)\end{array}$ & 91 & 127 & 0 & 33 & $73 \%$ & $100 \%$ \\
\hline $\begin{array}{l}\text { Giemsa alone } \\
(\mathrm{n}=251)\end{array}$ & 101 & 127 & 0 & 23 & $81 \%$ & $100 \%$ \\
\hline
\end{tabular}

${ }^{\dagger}$ Hematoxylin and Eosin

Histology was the most sensitive method of detecting H. pylori among these patients. Giemsa staining showed a marginally better sensitivity when compared with H\&E. Urease test showed a moderate sensitivity and specificity while culture and serology had lower sensitivity. As histology was taken as the reference, both staining methods showed $100 \%$ specificity.
Serology and culture also showed very high specificity while urease test had the lowest specificity.

Table 2 summarizes the sensitivity and specificity of $\mathrm{H} \& \mathrm{E}$ and modified Giemsa alone in detecting $H$. pylori in antrum and corpus compared with the gold standard.

Table 2: Sensitivity and specificity of $H$. pylori positivity in antrum and corpus when compared with the "gold standard" in which two staining methods are taken together.

\begin{tabular}{lllllll}
\hline Test & $\begin{array}{l}\text { True } \\
\text { Positives }\end{array}$ & $\begin{array}{l}\text { True } \\
\text { Negatives }\end{array}$ & $\begin{array}{l}\text { False } \\
\text { Positives }\end{array}$ & $\begin{array}{l}\text { False } \\
\text { Negatives }\end{array}$ & Sensitivity & Specificity \\
\hline $\begin{array}{l}\text { H. pylori in antrum } \\
\text { (H\&E }\end{array}$ & 88 & 127 & 0 & 36 & $71 \%$ & $100 \%$ \\
$\begin{array}{l}\text { H. pylone) } \\
\text { (H\&E } E^{\dagger} \text { alone) }\end{array}$ & 35 & 127 & 0 & 89 & $28 \%$ & $100 \%$ \\
$\begin{array}{l}\text { H. pylori in antrum } \\
\text { (Giemsa alone) }\end{array}$ & 98 & 127 & 0 & 26 & $79 \%$ & $100 \%$ \\
$\begin{array}{l}\text { H. pylori in corpus } \\
\text { (Giemsa alone) }\end{array}$ & 91 & 127 & 0 & 33 & $73 \%$ & $100 \%$ \\
\hline
\end{tabular}

${ }^{\dagger}$ Hematoxylin and Eosin 
Giemsa stain in biopsies obtained from either antrum or corpus showed $73-79 \%$ sensitivity. Similar sensitivity was observed with H\&E stain when used in biopsies obtained from antrum. Mucosal biopsies from corpus stained with $\mathrm{H} \& \mathrm{E}$ showed the lowest sensitivity (28\%).

Of the four tests, histology was the most costly test. It costs around 4 - 5 US Dollars (USD) per test for consumables. Approximately 2 hours was required to complete the test for one patient. This includes preparations for processing, embedding, section cutting, staining with both H\&E and Giemsa and the examination and reporting of the slides by the histopathologist. The estimated cost for man power for one test was 2 USD and therefore the total cost of the test was between 6-7 USD.

Serology was the second most expensive test. On average the consumable cost for one serology test was 3.8 USD. Of the total time required for the test, approximately one hour utilizes direct involvement of man power such as the time required to preparation and dilution of the sample, pipetting the sample and other ingredients and to read the test. Therefore the estimated cost for man power per one serology test was approximately 1 USD and the total cost was 4.8 USD.

Culture was in the third place when the total cost was compared. But it was more time consuming than serology. It required around 2.9 USD for consumables to perform culture for one patient. On average it required about 1.5 hours to perform culture. This included the time required for media preparation, autoclaving, preparing the culture plates, inoculation and colony identification. The approximate cost for man power to perform one culture test was 1.6 USD. The total cost was 4.5 USD.

Urease test was the cheapest of all tests. Since we used the home-made urease solution it required less than 0.2 USD to prepare $100 \mathrm{ml}$ of the solution and the time spent was approximately one hour. This included the time for weighing ingredients, preparing the solution and adjusting the $\mathrm{pH}$ of the final solution. Even though only $1 \mathrm{ml}$ of this solution was required per test the approximate cost for man power to perform one urease test was 1 USD. Hence the total cost for urease test was 1.2 USD.

The costs for the four diagnostic tests are summarized in table 3 .
Table 3: Cost comparisons of histology, serology, culture and urease test.

\begin{tabular}{lccc}
\hline Test & $\begin{array}{c}\text { Cost of } \\
\text { Consumables } \\
\left(\mathbf{U S D}^{\dagger}\right)\end{array}$ & $\begin{array}{c}\text { Cost of } \\
\text { Manpower } \\
\left(\text { USD }^{\dagger}\right)\end{array}$ & $\begin{array}{c}\text { Total } \\
\text { Cost } \\
\left(\text { USD }^{\dagger}\right)\end{array}$ \\
\hline Histology & $4-5$ & 2 & $6-7$ \\
Serology & 3.8 & 1 & 4.8 \\
Culture & 2.9 & 1.6 & 4.5 \\
Urease test & $<0.2$ & 1 & 1.2 \\
\hline
\end{tabular}

${ }^{\dagger}$ United States Dollar

\section{Discussion}

There is no gold standard for the detection of $H$. pylori in clinical practice and the choice of the test varies between clinicians. We considered histology as the gold standard in our study. Modified Giemsa stain was used as the special stain to improve the sensitivity and specificity of diagnosing $\mathrm{H}$. pylori as recommended by the updated Sydney system (14). When compared with the gold standard, sensitivity of $\mathrm{H} \& \mathrm{E}$ alone was $73.4 \%$ and the sensitivity of Giemsa alone was $81.5 \%$. As the chosen gold standard was based on these two techniques, both stains, as expected, showed $100 \%$ specificity. Our findings support the fact that Modified Giemsa is a reliable, less technically demanding and easily reproducible amongst many available staining techniques for $H$. pylori (15).

Our findings indicate that histology is the most expensive test despite its high sensitivity (97\%) and specificity (100\%). Many western countries, where the prevalence of infection is low, do not use histology in their routine clinical practice. The need of invasive endoscopy, time involved with preparation of biopsy specimens and cost are considered as limitations. However, in Sri Lanka endoscopy and histology are been done free of charge in most of the government owned tertiary care hospitals around the country. Hence, the cost factor has not limited the diagnostic utility of this investigation.

The rapid urease test when compared with histology, showed relatively low specificity and sensitivity, $32 \%$ and $68 \%$ respectively. This is not in keeping 
with many other studies where the rapid urease test has reported high specificity and sensitivity $(16,17)$.

In our study there were 31 patients with false positive urease test. Since the urease solution was sterile and the sterility was maintained during the biopsy procedure, contamination is an unlikely explanation. Recent findings show that hypochlorhydric patients could harbor many urease-positive bacteria in gastric mucosa other than H. Pylori (18). The strong urease activity they posses is suggested to be responsible for false positive results.

There were 67 false negative urease tests. Since many studies have shown the sensitivity of urease test to be around $80-90 \%$, the sensitivity we observed is lower. The sensitivity can vary with the site chosen for biopsy due to patchy distribution of the infection. In one study the gastric angle site was positive in $100 \%$, while the prepyloric and corpus sites were positive in $87 \%$ and $84.4 \%$, respectively (19). Hence a false negative test could occur due to sampling error. Satarasinghe, et al in 2000 have postulated that Sri Lankan H. pylori strains are different from strains found elsewhere that they have shorter survival and they produce inconspicuous rapid urease results (20). Some of the tests carried out among Sri Lankans have shown late urease test positivity. However urease test was the cheapest and the easiest to perform of the four tests

Infection with $H$. pylori induces several antibodies; anti $H$. pylori IgG, IgA and less frequently IgM. IgM can be detected shortly after infection is acquired and IgG and IgA antibodies indicate chronic infection. In most studies the prevalence of infection has been determined by IgG-type antibodies. Detection of serum $\operatorname{IgA}$ or $\operatorname{IgM}$ is known to have poor discriminatory value when compared with serum IgG. Some investigators have reported a subset of patients who are positive for IgA but negative for IgG antibodies for H.pylori.

Generally the serological prevalence is comparable with the prevalence of infection detected by other methods. The two cases with false positive results we observed may have had a previous infection with $H$. pylori which resulted in persistently elevated antibody levels. Due to the patchy distribution of $H$. pylori a sampling error could also have been the cause for the false positives. Similar picture has been observed in elderly people where the progression of atrophic gastritis has spontaneously eliminated the organism while there are detectable antibody levels in serum (21).

The false negative results could be due to many reasons. There are reported instances where certain test kits were being unable to detect the infection in certain populations (22). Furthermore, the prevalence of IgG antibodies to $H$. pylori was lower in elderly people when compared with those above 40 yrs of age (23).

Similar to positive histology, culture of bacterium which is urease, oxidase and catalase positive is a definitive proof of $H$ pylori infection. However, the ability to isolate the organism from infected subjects varies widely between laboratories and makes it the most technically demanding $H$. pylori diagnostic test. Biopsy specimens must be rapidly transferred to the laboratory in chilled transport medium, where upon the receipt, the sample is ground or minced to produce a homogenate which is inoculated on to freshly prepared media. Only when these fastidious requirements are observed does culture yields positive results.

Even after fulfilling all these requirements the sensitivity of culture varies between laboratories. Moayyedi, and Dixon have performed H. pylori culture with the sensitivity and specificity of $90.5 \%$ and $99.2 \%$ respectively (24). In one study that compared eight different methods for detection of H.pylori, culture revealed $55.9 \%$ sensitivity with $100 \%$ specificity (25).

Previous drug therapy such as antibiotics, omeprazole or Bismuth containing drugs during three months prior to culture are likely to give negative results $(26,27)$. Recent users of proton pump inhibitors were not excluded in our study and this may have contributed to negative cultures. Furthermore, commonly used non-steroidal antiinflammatory drugs can inhibit the growth of H. pylori in vitro (28).

The value of culture as a diagnostic test in Sri Lanka is questionable. Use of culture is mostly confined to antimicrobial susceptibility testing of $H$. pylori obtained from gastric biopsies prior to the first therapy and after the first treatment failure since macrolide resistance in this pathogen is considered a main reason for failure of antibiotic eradication therapy. 
According to our data, histology from multiple biopsies representing different areas of the stomach increases the detection of Hpylori. If endoscopist has a practical limitation in obtaining multiple biopsies, single biopsy obtained from antrum and stained with Modified Giemsa gives acceptable sensitivity to detect the organism (table 2).

There were certain limitations in direct and indirect cost calculations. For histology when calculating the reagent volumes required for staining techniques, total calculated volume was more than the true volume that was required. The reason is that once we perform a staining procedure, we do not wait till the reagent volume is completely over. Instead we refilled the deficit amount of reagent when required. So our estimate tends to be more than the actual value. Furthermore in all four diagnostic tests there were varying time periods with minimal personnel involvement e.g. tissue processing time in histology, incubation periods in serology and culture. This has not being taken into consideration when calculating indirect costs. So the actual number of hours taken by the test was more than the time we estimated.

In this study, as instructed by the manufacturer, plasma instead of serum was used. Assurance was given by the manufacturer that both plasma and serum would give same results when using these kits (29).

\section{Acknowledgements}

Dr. Hasanjaya Gunawardena, Consultant Gastroenterological Surgeon, for granting permission and providing access to enroll the patients for the study at his Endoscopy Unit, Teaching Hospital Karapitiya, Galle, Sri Lanka.

Technical staff of Departments of Pathology, Microbiology, Biochemistry and Nuclear Medicine Unit, Faculty of Medicine, Galle, Sri Lanka for the technical support.

University Research Grant, University of Ruhuna, Sri Lanka, for providing financial support for the study.

No conflicts of interest disclosed.

\section{References}

1. Prasad S, Mathan M, Chandy G. et al. Prevalence of Helicobacter pylori in Southern Indian controls and patients with gastroduodenal disease. Journal of Gastroenterology and Hepatology 1994; 9: 501-6.

2. Kazi JI, Jafarey NA, Alam SM. et al. Association of Helicobacter pylori with acid peptic disease in Karachi. Journal of Pakistan Medical Association 1990; 40: 240-1.

3. Mahalanabis D, Rahman MM, Sarker SA. et al. Helicobacter pylori infection in the young in Bangladesh: prevalence, socioeconomic and nutritional aspects. International Journal of Epidemiology 1996; 25: 894-8.

4. Fernando D. Helicobacter pylori and peptic ulcer disease in Sri Lanka. Ceylon Medical Journal 1992; 37: 15-7.

5. De Silva M. Prevalence of Helicobacter pylori infection in patients with functional dyspepsia. Ceylon Medical Journal 1999; 44: 118-9.

6. Fernando N, Holton J, Vaira D, De Silva M, Fernando D. Prevalence of Helicobacter pylori in Sri Lanka as Determined by PCR. Journal of Clinical Microbiology 2002; 40: 2675-6.

7. Dent JC, McNulty CA. Evaluation of a new selective medium for Campylobacter pylori. European Journal of Clinical Microbiology and Infectious Diseases 1988; 7 : 555-8.

8. Barrow GI, Feltham RKA. Cowan and Steel's Manual for the identification of Medical Bacteria. Cambridge: Cambridge University Press, 1993.

9. McKay GS. Gram stain modified to improve color contrast. Journal of Clinical Pathology 1970; 23: 191.

10. McNulty CA, Dent JC, Uff JS, Gear MW, Wilkinson SP. Detection of Campylobacter pylori by the biopsy urease test: an assessment in 1445 patients. Gut 1989; 30: 1058-62.

11. Gray SF, Wyatt JI, Rathbone BJ. Simplified techniques for identifying Campylobacter pyloridis. Journal of Clinical Pathology 1986; 39: 1279.

12. Wong BC, Wong WM, Wang WH. et al. An evaluation of invasive and non-invasive tests for the diagnosis of Helicobacter pylori infection in Chinese. Alimentary Pharmacology and Therapeutics 2001; 15: 505-11.

13. Barthel JS, Everett ED. Diagnosis of Campylobacter pylori infections: the "gold standard" and the alternatives. Reviews of Infectious Diseases 1990; 12 (Suppl. 1): S10714. 
14. Dixon MF, Genta RM, Yardley JH, Correa P. Classification and grading of gastritis. The updated Sydney System. International Workshop on the Histopathology of Gastritis, Houston 1994. American Journal of Surgical Pathology 1996; 20: 1161-81.

15. Rotimi O, Cairns A, Gray S, Moayyedi P, Dixon MF. Histological identification of Helicobacter pylori: comparison of staining methods. Journal of Clinical Pathology 2000; 53: 756-9.

16. Haruma K, Okamoto S, Sumii K. et al. Helicobacter pylori infection and gastroduodenal disease: a comparison of endoscopic findings, histology, and urease test data. Hiroshima Journal of Medical Sciences 1992; 41: 65-70.

17. Bermejo F, Boixeda D, Gisbert JP. et al. Rapid urease test utility for Helicobacter pylori infection diagnosis in gastric ulcer disease. Hepatogastroenterology 2002; 49: 572-5.

18. Brandi G, Biavati B, Calabrese C et al. Urease-positive bacteria other than Helicobacter pylori in human gastric juice and mucosa. American Journal of Gastroenterology 2006; 101: 1756-61.

19. Woo JS, el-Zimaity HM, Genta RM, Yousfi MM, Graham DY. The best gastric site for obtaining a positive rapid urease test. Helicobacter 1996; 1: 256-9.

20. Satarasinghe RL, Jayamaha DH, Vidyathilake HMS, Siriwardene S. Do our Helicobacter pylori differ from those found in the west, in their ability to produce urease enzyme? - A preliminary observation. Journal of the Ceylon College of Physicians 2000;33: 121-3.

21. Fox JG, Correa P, Taylor NS. et al. Campylobacter pyloriassociated gastritis and immune response in a population at increased risk of gastric carcinoma. American Journal of Gastroenterology 1989; 84: 775-81.

22. Miwa H, Kikuchi S, Ohtaka K et al. Insufficient diagnostic accuracy of imported serological kits for Helicobacter pylori infection in Japanese population. Diagnostic Microbiology and Infectious Diseases 2000; 36: 95-9.
23. Asaka M, Sugiyama T, Nobuta A, Kato M, Takeda H, Graham DY. Atrophic gastritis and intestinal metaplasia in Japan: results of a large multicenter study. Helicobacter 2001; 6: 294-9.

24. Moayyedi P, Dixon MF. Any role left for invasive tests? Histology in clinical practice. Gut 1998; 43(Suppl.1): S515.

25. Kullavanijaya $\mathrm{P}$, Thong-Ngam D, Hanvivatvong O, Nunthapisud P, Tangkijvanich P, Suwanagool P. Analysis of eight different methods for the detection of Helicobacter pylori infection in patients with dyspepsia. Journal of Gastroenterology and Hepatology 2004; 19: 1392-6.

26. Mirshahi F, Fowler G, Patel A, Shaw G. Omeprazole may exert both a bacteriostatic and a bacteriocidal effect on the growth of Helicobacter pylori (NCTC 11637) in vitro by inhibiting bacterial urease activity. Journal of Clinical Pathology 1998; 51:220-4.

27. Kung NN, Sung JJ, Yuen NW. et al. One-week ranitidine bismuth citrate versus colloidal bismuth subcitrate-based anti-Helicobacter triple therapy: a prospective randomized controlled trial. American Journal of Gastroenterology 1999; 94: 721-4.

28. Shirin H, Moss SF, Kancherla S. et al. Non-steroidal antiinflammatory drugs have bacteriostatic and bactericidal activity against Helicobacter pylori. Journal of Gastroenterology and Hepatology 2006; 21: 1388-93.

29. Lee AS, Finkielman JD, Peikert T. et al. Agreement of antineutrophil cytoplasmic antibody measurements obtained from serum and plasma. Clinical and Experimental Immunology 2006; 146: 15-20. 